\title{
Effect of Salinity on Seed Germination and Seedling Development of Soybean Genotypes
}

\author{
Ourania I Pavli*, Chrysanthi Foti, Georgia Skoufogianni, Georgia Karastergiou, Asimo Panagou and Ebrahim M \\ Khah \\ Department of Agriculture, Crop Production and Rural Environment, University of Thessaly, Greece
}

Submission: February 08, 2021; Published: March 01, 2021

*Corresponding author: Ourania I Pavli, Department of Agriculture, Crop Production and Rural Environment, School of Agricultural Sciences, University of Thessaly, Volos, Greece

\begin{abstract}
Soybean is one of the most important staple legume crops worldwide due to its high protein and oil content as well as its nitrogen fixing ability. Soybean is classified as moderate salt sensitive crop, with salinity causing adverse effects on all developmental stages, including seed germination and post-germinative growth. This study aimed at determining seed germination and seedling growth potential under salt stress conditions as a short-cut approach to identify salt tolerant genotypes at early growth stages. To this direction, nine commercial and pre-commercial varieties, whose adaptation to increased soil salinity is unknown, were imposed to salt stress using solutions differing in $\mathrm{NaCl}$ concentration $(50,100$, $200 \mathrm{mM} \mathrm{NaCl}$, while non-stressed plants served as controls. Evaluation of tolerance was performed on the basis of germination percentage, seed water absorbance, seedling water content, root and shoot length and number of seedlings with abnormal phenotype. Overall findings revealed that stress substantially affects all traits associated to seed germination and seedling growth, with the effects being analogous to the stress level applied, yet genotypes exhibited varying response to increased salinity. Overall data suggest that seed germination and seedling growth potential may be readily employed to reveal genetic variability related to salt tolerance in soybean germplasm. Such screening approach may serve for selecting suitable germplasm material at early growth stages, thus upgrading all relevant breeding procedures.
\end{abstract}

Keywords: Abiotic stress; Early selection; NaCl-induced salt stress; Salt tolerance; Screening method; Screening criteria

\section{Introduction}

Soybean (Glycine max L.) is among the most important legume crops, providing considerable amounts of protein and oil for human consumption, while improving soil properties through establishment of symbiotic associations with $\mathrm{N}$-fixing bacteria. Its cultivation, however, is often restricted by the crop's high input demands and the fact that the achievement of its yield potential is subjected to optimum agroclimatic conditions. Such limitations are further strengthened by the gradual reduction of available natural resources and degradation of agricultural land worldwide [1]. In view of the obvious need to conform with the criteria posed by sustainable agriculture, requiring the reduction of inputs, the development of soybean varieties with genetic resistance and yield stability under suboptimal agroclimatic conditions is the most efficient and cost-effective strategy to address an economic crop viability.

Abiotic stresses pose serious constraints to agricultural crop production worldwide, with salinity viewed as one of the most important both in terms of effects on plant growth and crop productivity [2]. The adverse effects of salinity primarily reflect the result of an extremely reduced osmotic potential and ion toxicity around the rhizosphere, leading to inhibition of water uptake by hairy roots and increased uptake of sodium $\left(\mathrm{Na}^{+}\right)$and chloride $\left(\mathrm{Cl}^{-}\right)$ions by plant cells, ultimately evidenced as ion imbalance and secondary oxidative stress [3-6]. Consequently, salinity stress hampers plant growth and development by altering essential metabolic processes such as $\mathrm{CO}_{2}$ assimilation, protein and oil synthesis $[7,8]$. It is worth noting however, that plant species vary tremendously in their ability to cope with salinity stress, with soybean being classified as moderately salt sensitive crop [9] with a salinity threshold of 5.0dS/m [10-12].

Routinely, crops' response to salinity stress is estimated on the basis of yield potential under salinity stress, while the emphasis is placed on determining the yield penalties attributed to soil salinity. Such an approach is rather laborious, time-consuming and often ineffective, mainly due to the complex genetic nature of salt tolerance traits as well as the limitations arising from obtaining homogeneous stress conditions in field trials. As an alternative, 
it has been proposed that salt tolerance at early growth stages is a heritable trait which, at the same time, provides an accurate estimation of growth and yield potential under salt conditions [13]. Recent studies underline that salinity stress during germination and early growth crucially affect crop growth [14], while inhibition and/or delay of seed germination and seedling growth due to salt stress is correlated with decreased yield in soybean [15-18]. In this context, this study aimed at determining seed germination and seedling growth potential under salinity stress conditions as a short-cut approach to identify salt tolerant soybean genotypes at early growth stages.

\section{Material and Methods}

\section{Plant material}

The genetic material consisted of nine commercial and precommercial soybean varieties, whose adaptation to increased soil salinity is not clarified. The germplasm under study included the varieties: PR92B63, PR92M35, PR92M22, PR91M10, ZORA, NEOPLANTA, P21T45, CELINA and ADONAI.

\section{Growth conditions and experimental design}

Seeds were initially surface-sterilized in 20\% hypochlorite/ $\mathrm{dH}_{2} \mathrm{O}$ solution, containing Tween-20, while gently mixing for $5 \mathrm{~min}$, and washed ( $4 \mathrm{x})$ with sterile $\mathrm{dH}_{2} \mathrm{O}$. Sterilized seeds were allowed to germinate in plastic trays containing solutions differing in $\mathrm{NaCl}$ concentration: i) $0 \mathrm{mM} \mathrm{NaCl}$, ii) $50 \mathrm{mM} \mathrm{NaCl}$, iii) $100 \mathrm{mM} \mathrm{NaCl}$ and iv) $200 \mathrm{mM} \mathrm{NaCl}$. Plants grown in distilled $\mathrm{H}_{2} \mathrm{O}(0 \mathrm{mM} \mathrm{NaCl})$ were included as controls. Plants were grown under controlled conditions $\left(25^{\circ} \mathrm{C}, 16 \mathrm{~h}\right.$ light/8h dark) for a period of 20 days.

The experimental layout was that of a completely random design with four replications, each consisting of 30 seeds. The experimental plots consisted of four rows, of which the two middle provided the material for the measurements.

\section{Measurements}

Variety performance was assessed on the basis of seed germination and seedling growth potential under salinity stress conditions. As evaluation criteria served the following parameters: germination percentage, seed water uptake, seedling water content, root and shoot length and number of seedlings with abnormal phenotype. All measurements were conducted when the radicle had a length of at least $2 \mathrm{~mm}$.

Germination Percentage (GP \%) was estimated at five time intervals $\left(3^{\text {rd }}-7^{\text {th }}\right.$ day $)$, according to the formula GP $=$ seeds germinated / total seeds x 100. Seed water absorbance (WU\%) was calculated at $5^{\text {th }}$ and $7^{\text {th }}$ day, following the formula $\mathrm{WU}(\%)=$ $\left(W_{2}-W_{1}\right) / W_{1} \times 100$, where $W_{1}=$ initial seed weight and $W_{2}=$ seed weight after water absorbance [19]. Seedling water content (WC $\%$ ) was determined at $7^{\text {th }}$ and $12^{\text {th }}$ day, according to the formula WC $(\%)=(F W-D W / F W) \times 100$, where FW = fresh weight and DW $=$ dry weight [20]. Measurements for shoot and root length $(\mathrm{cm})$ were conducted at $5^{\text {th }}, 8^{\text {th }}, 11^{\text {th }}$ and $15^{\text {th }}$ day. The number of seedlings with abnormal genotype, mainly referring to sprouts with spiral hypocotyl and dwarf primary root [21], was recorded throughout the period of observations.

For estimation of WU, the weight of twenty seeds (five per replication) was taken into account, while for shoot and root length as well as for FW and DW twenty seedlings (five from each replication) were used. DW was determined following incubation at $70{ }^{\circ} \mathrm{C}$, for 48 hours.

\section{Statistical analysis}

Data were analyzed by ANOVA according to the experimental design. The performance of genotypes was comparatively assessed within stress levels but also across stress levels, while the effect of salinity stress was comparatively assessed across genotypes. Differences between pair of means were assessed using the Student's LSD test ( $\mathrm{p} \leq 0.05$ ). All statistical analyses were performed using JMP software v.8 (SAS, Cary, NC, USA).

\section{Results}

Overall findings revealed that all traits related to germination and seedling growth were severely affected by salt stress, with the effects of stress gradually intensifying as salt concentration increased. It is worth noting however, that genotypes under study exhibited varying response to increased salinity.

Although germination potential was significantly affected at all stress levels in the entire set of genotypes, the reduction was in general analogous to the stress level applied (Table 1, Figure 1). The onset of germination was at the $3^{\text {rd }}$ day and was accompanied by significant variation both at treatment and genotype level. In the absence of stress, the higher GP was noted in varieties Neoplanta and Adonai, followed by PR92M22, Celina and Zora. At this time point, PR92B63, PR92M35, PR91M10 and P21T45 showed very low or zero GP values. Upon stress, a gradual decrease in GP was observed as $\mathrm{NaCl}$ concentration increased, thus leading to most profound decrease at $200 \mathrm{mM} \mathrm{NaCl}$. At all stress levels, varieties Neoplanta and Adonai presented the highest values for GP. Although initially ( $3^{\text {rd }}$ day) presented a significant decrease both at 100 and $200 \mathrm{mM} \mathrm{NaCl}$, from the $4^{\text {th }}$ day onwards Neoplanta and Adonai maintained a relatively high GP, yet even these varieties exhibited a significant decrease in GP at $200 \mathrm{mM} \mathrm{NaCl}$. In contrast, varieties PR92M22, Celina and Zora performed satisfactorily at $50 \mathrm{mM} \mathrm{NaCl}$ but showed a drastic decrease in GP at 100 and $200 \mathrm{mM} \mathrm{NaCl}$. On the other hand, varieties PR92B63, PR92M35, PR91M10 and P21T45 presented low values for GP both under control and stress conditions. Collectively, Neoplanta and Adonai appeared as the most tolerant varieties, with their superiority being evidenced both by their mean response across stress levels and their final GP (Neoplanta: GP $=75 \%(50 \mathrm{mM}$ and $100 \mathrm{mM}$ ) and 45\% (200mM), Adonai: GP=69,17\% (50mM), 64,17\% (100 $\mathrm{mM})$ and $27,50 \%(200 \mathrm{mM})$ at the $7^{\text {th }}$ day). Neoplanta's ability to retain a high $\mathrm{GP}$ even at $200 \mathrm{mM} \mathrm{NaCl}$, during the entire period of observation, is indicative of its potential to cope with severe salt stress conditions. 
Table 1: Germination percentage (GP \%) as affected by genotype (G) and $\mathrm{NaCl}$ concentration (C) at five different time intervals ( $3^{\text {rd }}$ to $7^{\text {th }}$ day) of salinity stress.

\begin{tabular}{|c|c|c|c|c|c|c|c|c|c|c|}
\hline \multirow{3}{*}{$\begin{array}{c}\text { Time (d) } \\
3\end{array}$} & \multirow{3}{*}{ Genotype (G) } & \multicolumn{8}{|c|}{$\mathrm{NaCl}$ Concentration $(\mathrm{mM})(\mathrm{C})$} & \\
\hline & & \multicolumn{2}{|c|}{$\mathbf{0}$} & \multicolumn{2}{|c|}{50} & \multicolumn{2}{|c|}{100} & \multicolumn{2}{|c|}{200} & \multirow{2}{*}{ MEAN ( G ) } \\
\hline & & & & & & & & & & \\
\hline & PR92B63 & 0,00 & $\mathrm{c}$ & 0,00 & $\mathrm{c}$ & 0,00 & c & 0,00 & $\mathrm{~b}$ & $0,00 \mathrm{e}$ \\
\hline & PR92M35 & 0,83 & $\mathrm{c}$ & 0,00 & $\mathrm{c}$ & 0,00 & c & 0,00 & $\mathrm{~b}$ & $0,21 \mathrm{e}$ \\
\hline & PR92M22 & 20,00 & $\mathrm{~b}$ & 8,33 & $\mathrm{c}$ & 2,50 & $\mathrm{bc}$ & 0,00 & $\mathrm{~b}$ & $7,71 \mathrm{c}$ \\
\hline & PR91M10 & 0,00 & $\mathrm{c}$ & 0,00 & $\mathrm{c}$ & 0,00 & c & 0,00 & $\mathrm{~b}$ & $0,00 \mathrm{e}$ \\
\hline & Zora & 15,83 & $\mathrm{~b}$ & 2,50 & $\mathrm{c}$ & 0,00 & c & 0,00 & $\mathrm{~b}$ & $4,58 \mathrm{~cd}$ \\
\hline & Neoplanta & 71,67 & $\mathrm{a}$ & 52,50 & $\mathrm{a}$ & 17,50 & a & 5,00 & $\mathrm{a}$ & $36,67 a$ \\
\hline & P21T45 & 3,33 & $\mathrm{c}$ & 1,67 & $\mathrm{c}$ & 0,83 & $\mathrm{bc}$ & 0,00 & $\mathrm{~b}$ & $1,46 \mathrm{de}$ \\
\hline & Celina & 17,50 & $\mathrm{~b}$ & 8,33 & $\mathrm{c}$ & 5,00 & $\mathrm{bc}$ & 2,50 & $\mathrm{ab}$ & $8,33 c$ \\
\hline & Adonai & 61,67 & $\mathrm{a}$ & 40,83 & $\mathrm{~b}$ & 10,00 & $\mathrm{ab}$ & 0,00 & $\mathrm{~b}$ & $28,12 b$ \\
\hline & SED & 3,01 & & 2,69 & & 2,77 & & 0,87 & & $\operatorname{SED}(G)=1,25$ \\
\hline & MEAN ( C ) & 21,2 & $\mathrm{a}$ & 12,68 & $\mathrm{~b}$ & 3,98 & $\mathrm{c}$ & 0,83 & $\mathrm{~d}$ & $\operatorname{SED}(\mathrm{C})=0,83$ \\
\hline \multirow[t]{12}{*}{4} & & & & & & & & & & MEAN ( G ) \\
\hline & PR92B63 & 0,00 & $\mathrm{c}$ & 0,00 & $\mathrm{~d}$ & 0,00 & c & 0,00 & $\mathrm{c}$ & $0,00 \mathrm{e}$ \\
\hline & PR92M35 & 17,50 & $\mathrm{c}$ & 4,17 & $\mathrm{~d}$ & 0,00 & c & 0,00 & $\mathrm{c}$ & $5,41 \mathrm{de}$ \\
\hline & PR92M22 & 49,17 & $\mathrm{~b}$ & 37,50 & $\mathrm{~b}$ & 17,50 & $\mathrm{bc}$ & 21,67 & $\mathrm{ab}$ & $31,46 \mathrm{~b}$ \\
\hline & PR91M10 & 0,83 & $\mathrm{c}$ & 0,00 & $\mathrm{~d}$ & 0,00 & c & 0,00 & $\mathrm{c}$ & $0,21 \mathrm{e}$ \\
\hline & Zora & 43,33 & $\mathrm{~b}$ & 16,67 & $\mathrm{~cd}$ & 13,33 & $\mathrm{bc}$ & 0,00 & $\mathrm{c}$ & $18,33 \mathrm{c}$ \\
\hline & Neoplanta & 80,00 & $\mathrm{a}$ & 70,00 & $\mathrm{a}$ & 65,00 & a & 31,67 & $\mathrm{a}$ & $61,67 a$ \\
\hline & P21T45 & 17,50 & $\mathrm{c}$ & 11,67 & $\mathrm{~d}$ & 9,17 & $\mathrm{bc}$ & 0,83 & $\mathrm{c}$ & $9,79 \mathrm{~cd}$ \\
\hline & Celina & 44,17 & $\mathrm{~b}$ & 34,17 & $\mathrm{bc}$ & 30,00 & $\mathrm{~b}$ & 17,50 & $\mathrm{~b}$ & $31,46 \mathrm{~b}$ \\
\hline & Adonai & 80,00 & $\mathrm{a}$ & 60,00 & $\mathrm{a}$ & 57,50 & a & 11,67 & $\mathrm{~b}$ & $52,29 a$ \\
\hline & SED & 7,47 & & 5,47 & & 6,87 & & 3,09 & & $\operatorname{SED}(G)=2,98$ \\
\hline & MEAN ( C ) & 36,94 & $\mathrm{a}$ & 26,02 & $\mathrm{~b}$ & 21,38 & $\mathrm{~b}$ & 9,26 & $\mathrm{c}$ & $\operatorname{SED}(C)=1,99$ \\
\hline \multirow[t]{12}{*}{5} & & & & & & & & & & MEAN ( G ) \\
\hline & PR92B63 & 0,00 & $\mathrm{~d}$ & 0,00 & $\mathrm{e}$ & 0,00 & d & 0,00 & $\mathrm{c}$ & $0.00 \mathrm{e}$ \\
\hline & PR92M35 & 18,33 & $\mathrm{~d}$ & 7,50 & de & 1,67 & $\mathrm{~cd}$ & 0,83 & $\mathrm{c}$ & 7.08ef \\
\hline & PR92M22 & 54,17 & bc & 40,83 & $\mathrm{~b}$ & 21,67 & $\mathrm{bc}$ & 24,17 & $\mathrm{~b}$ & $35.21 \mathrm{c}$ \\
\hline & PR91M10 & 1,67 & $\mathrm{~d}$ & 0,00 & $\mathrm{e}$ & 0,00 & d & 0,00 & $\mathrm{c}$ & $0.42 \mathrm{f}$ \\
\hline & Zora & 45,00 & $\mathrm{c}$ & 19,17 & $\mathrm{~cd}$ & 17,50 & bcd & 0,83 & $\mathrm{c}$ & $20.62 \mathrm{~d}$ \\
\hline & Neoplanta & 80,00 & $\mathrm{a}$ & 70,00 & $\mathrm{a}$ & 69,17 & a & 35,83 & $\mathrm{a}$ & $63.75 a$ \\
\hline & P21T45 & 20,00 & $\mathrm{~d}$ & 14,17 & de & 10,83 & bcd & 1,67 & $\mathrm{c}$ & $11.67 \mathrm{e}$ \\
\hline & Celina & 44,17 & $\mathrm{c}$ & 33,33 & $\mathrm{bc}$ & 30,83 & $\mathrm{~b}$ & 20,00 & $\mathrm{~b}$ & $32.08 c$ \\
\hline & Adonai & 76,67 & $\mathrm{ab}$ & 61,67 & $\mathrm{a}$ & 60,00 & a & 13,33 & $\mathrm{~b}$ & $59.92 \mathrm{a}$ \\
\hline & SED & 6,83 & & 5,63 & & 6,00 & & 3,45 & & $\operatorname{SED}(G)=2,81$ \\
\hline & MEAN ( C ) & 37,78 & $\mathrm{a}$ & 27,41 & $\mathrm{~b}$ & 23,52 & $\mathrm{~b}$ & 10,74 & $\mathrm{c}$ & $\operatorname{SED}(C)=1,87$ \\
\hline \multirow[t]{4}{*}{6} & & & & & & & & & & MEAN ( G ) \\
\hline & PR92B63 & 0,00 & $\mathrm{~g}$ & 0,00 & $\mathrm{f}$ & 0,00 & c & 0,00 & $\mathrm{c}$ & $0,00 \mathrm{e}$ \\
\hline & PR92M35 & 24,17 & ef & 10,00 & ef & 3,33 & c & 1,67 & $\mathrm{c}$ & $7,79 d$ \\
\hline & PR92M22 & 60,83 & bc & 52,50 & bc & 25,00 & $\mathrm{~b}$ & 26,67 & $\mathrm{~b}$ & $41,50 \mathrm{~b}$ \\
\hline
\end{tabular}


International Journal of Environmental Sciences \& Natural Resources

\begin{tabular}{|c|c|c|c|c|c|c|c|c|c|c|}
\hline & PR91M10 & 1,67 & $\mathrm{fg}$ & 0,00 & $\mathrm{f}$ & 0,00 & $\mathrm{c}$ & 0,00 & c & $0,42 \mathrm{e}$ \\
\hline & Zora & 47,50 & $\mathrm{~cd}$ & 22,50 & de & 19,17 & $\mathrm{bc}$ & 4,17 & c & $23,33 c$ \\
\hline & Neoplanta & 83,33 & $a b$ & 74,17 & $\mathrm{a}$ & 72,50 & $\mathrm{a}$ & 40,83 & $\mathrm{a}$ & $67,71 \mathrm{a}$ \\
\hline & P21T45 & 25,83 & de & 19,17 & ef & 14,17 & $\mathrm{bc}$ & 4,17 & c & $15,83 \mathrm{~cd}$ \\
\hline & Celina & 48,33 & $\mathrm{~cd}$ & 40,00 & $\mathrm{~cd}$ & 30,83 & $\mathrm{~b}$ & 22,50 & $\mathrm{~b}$ & $35,41 b$ \\
\hline & Adonai & 85,00 & $\mathrm{a}$ & 66,67 & $a b$ & 63,33 & $\mathrm{a}$ & 22,50 & b & $59,37 \mathrm{a}$ \\
\hline & SED & 6,88 & & 6,17 & & 6,02 & & 3,97 & & $\operatorname{SED}(G)=2,93$ \\
\hline & MEAN ( C ) & 41,85 & $\mathrm{a}$ & 31,67 & $\mathrm{~b}$ & 25,37 & $\mathrm{c}$ & 13,61 & d & $\operatorname{SED}(C)=1,95$ \\
\hline \multirow[t]{12}{*}{7} & & & & & & & & & & MEAN ( G ) \\
\hline & PR92B63 & 0,00 & e & 0,00 & e & 0,00 & $\mathrm{c}$ & 0,00 & c & $0,00 \mathrm{e}$ \\
\hline & PR92M35 & 26,67 & $\mathrm{~cd}$ & 12,50 & de & 3,33 & c & 3,33 & c & $11,46 \mathrm{~d}$ \\
\hline & PR92M22 & 58,33 & $\mathrm{~b}$ & 60,83 & $\mathrm{ab}$ & 30,00 & $\mathrm{~b}$ & 29,17 & b & $44,58 b$ \\
\hline & PR91M10 & 2,50 & de & 0,83 & e & 0,00 & c & 0,00 & c & $0,83 \mathrm{e}$ \\
\hline & Zora & 47,50 & $\mathrm{bc}$ & 25,83 & $\mathrm{~cd}$ & 20,83 & $\mathrm{bc}$ & 5,83 & c & $25,00 \mathrm{c}$ \\
\hline & Neoplanta & 85,00 & $\mathrm{a}$ & 75,00 & a & 75,00 & $\mathrm{a}$ & 45,00 & a & $70,00 \mathrm{a}$ \\
\hline & P21T45 & 28,33 & c & 20,83 & cde & 17,50 & $\mathrm{bc}$ & 4,17 & c & $17,71 \mathrm{~cd}$ \\
\hline & Celina & 45,83 & $\mathrm{bc}$ & 40,83 & bc & 32,50 & $\mathrm{~b}$ & 25,00 & $\mathrm{~b}$ & $36,04 b$ \\
\hline & Adonai & 85,83 & $\mathrm{a}$ & 69,17 & a & 64,17 & $\mathrm{a}$ & 27,50 & $\mathrm{~b}$ & $61,67 a$ \\
\hline & SED & 7,29 & & 6,22 & & 6,32 & & 4,23 & & $\operatorname{SED}(G)=3,06$ \\
\hline & MEAN ( C ) & 42,22 & $\mathrm{a}$ & 33,98 & $\mathrm{~b}$ & 27,04 & c & 15,56 & d & $\operatorname{SED}(C)=2,04$ \\
\hline
\end{tabular}

${ }^{*}$ At each time interval (days), values followed by the same letter, within each factor, are not significantly different according to LSD (P $\left.\leq 0.05\right)$.
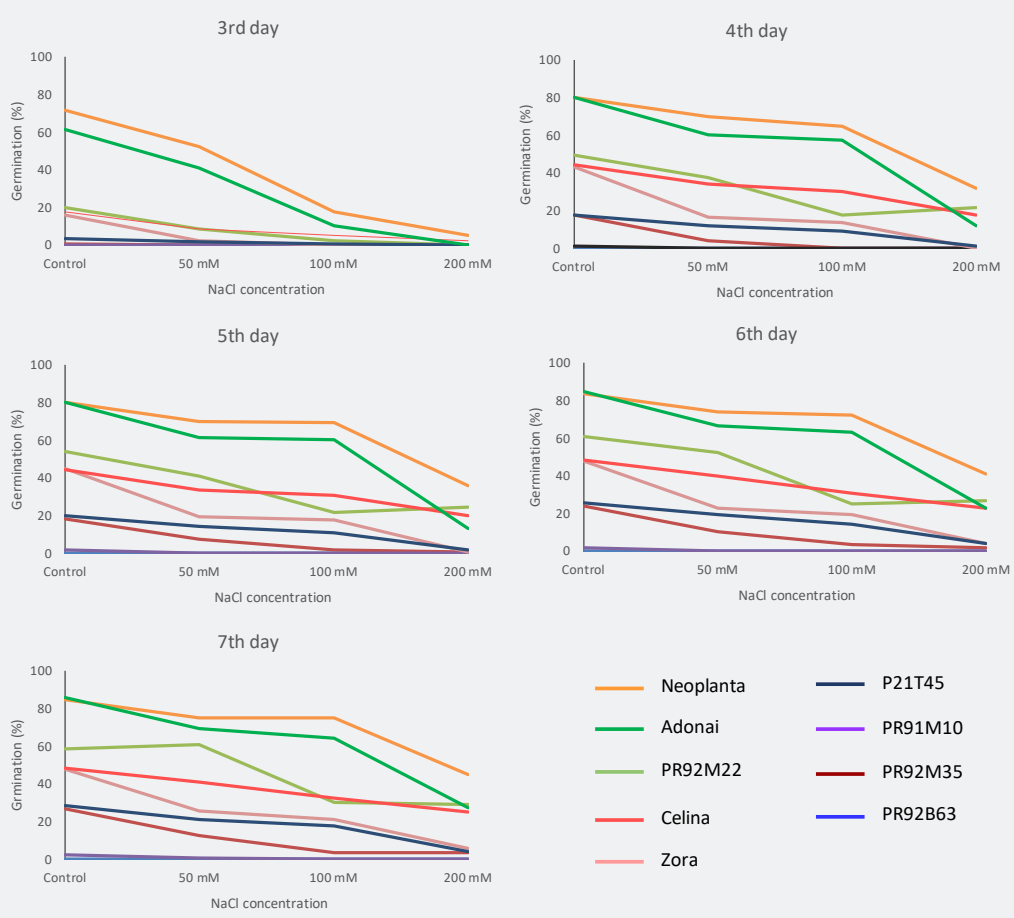

Figure 1: Germination percentage (\%) of nine soybean genotypes at different levels of salinity stress and at five time intervals. 
As far as WU is concerned, it was not substantially affected by salinity stress, while simultaneously the effects of stress were not well correlated to the level applied (Table 2), thus suggesting that WU does not provide an accurate screening criterion for salt tolerance. In contrast, salt stress significantly affected WC, with the effects of stress being in most cases proportional to the level applied (Table 3) as evidenced by the mean values of stress levels across genotypes. Despite differences observed among genotypes and stress levels, the final WC values of genotypes did not differ at $200 \mathrm{mM} \mathrm{NaCl}$.

Table 2: Seed water absorbance (WU \%) as affected by genotype (G) and $\mathrm{NaCl}$ concentration $(\mathrm{C})$ at $5^{\text {th }}$ and $7^{\text {th }}$ day of salinity stress.

\begin{tabular}{|c|c|c|c|c|c|c|c|c|c|c|}
\hline \multirow{3}{*}{$\begin{array}{c}\text { Time (d) } \\
5\end{array}$} & \multirow{3}{*}{ Genotype (G) } & \multicolumn{8}{|c|}{ NaCl Concentration (mM) ( C) } & \multirow[b]{3}{*}{ MEAN ( G ) } \\
\hline & & \multicolumn{2}{|c|}{$\mathbf{0}$} & \multicolumn{2}{|c|}{50} & \multicolumn{2}{|c|}{100} & \multicolumn{2}{|c|}{200} & \\
\hline & & & & & & & & & & \\
\hline & PR92B63 & 59,40 & $\mathrm{~b}$ & 57,59 & bc & 59,73 & $\mathrm{a}$ & 56,03 & $\mathrm{a}$ & $58,19 \mathrm{abc}$ \\
\hline & PR92M35 & 54,79 & $\mathrm{~b}$ & 57,30 & $\mathrm{bc}$ & 54,69 & $\mathrm{a}$ & 55,04 & $\mathrm{a}$ & $55,45 c$ \\
\hline & PR92M22 & 58,70 & $\mathrm{~b}$ & 59,08 & $a b c$ & 56,72 & $\mathrm{a}$ & 56,63 & $\mathrm{a}$ & $57,78 \mathrm{bc}$ \\
\hline & PR91M10 & 54,46 & $\mathrm{~b}$ & 54,46 & c & 56,52 & $\mathrm{a}$ & 56,31 & $\mathrm{a}$ & $55,44 \mathrm{c}$ \\
\hline & Zora & 57,46 & $\mathrm{~b}$ & 58,51 & $\mathrm{bc}$ & 56,71 & $\mathrm{a}$ & 57,68 & $\mathrm{a}$ & $57,59 \mathrm{bc}$ \\
\hline & Neoplanta & 68,09 & a & 66,94 & a & 59,75 & $\mathrm{a}$ & 54,25 & $\mathrm{a}$ & $62,26 a$ \\
\hline & P21T45 & 57,25 & $\mathrm{~b}$ & 58,47 & bc & 55,52 & $\mathrm{a}$ & 56,38 & $\mathrm{a}$ & $56,90 \mathrm{c}$ \\
\hline & Celina & 59,58 & $\mathrm{~b}$ & 56,45 & $\mathrm{bc}$ & 60,52 & $\mathrm{a}$ & 55,08 & $\mathrm{a}$ & $57,91 \mathrm{abc}$ \\
\hline & Adonai & 66,97 & a & 63,26 & $\mathrm{ab}$ & 58,51 & $\mathrm{a}$ & 57,18 & $\mathrm{a}$ & $61,48 \mathrm{ab}$ \\
\hline & SED & 2,15 & & 2,47 & & 2,19 & & 3,98 & & $\operatorname{SED}(G)=1,40$ \\
\hline & MEAN ( C ) & 59,63 & a & 59,12 & a & 57,63 & $\mathrm{ab}$ & 56,07 & $\mathrm{~b}$ & $\operatorname{SED}(C)=0,99$ \\
\hline \multirow[t]{12}{*}{7} & & & & & & & & & & MEAN ( G ) \\
\hline & PR92B63 & 52,62 & $\mathrm{e}$ & 54,16 & $\mathrm{~b}$ & 60,01 & $\mathrm{a}$ & 59,62 & $\mathrm{a}$ & $56,60 \mathrm{~d}$ \\
\hline & PR92M35 & 60,46 & cde & 38,67 & c & 46,49 & $\mathrm{~b}$ & 45,98 & $\mathrm{c}$ & $47,90 \mathrm{e}$ \\
\hline & PR92M22 & 68,34 & $\mathrm{abc}$ & 59,56 & $\mathrm{ab}$ & 58,25 & $\mathrm{a}$ & 53,09 & $\mathrm{~b}$ & $59,81 \mathrm{bcd}$ \\
\hline & PR91M10 & 56,72 & de & 60,37 & $\mathrm{ab}$ & 56,59 & $\mathrm{ab}$ & 57,93 & $\mathrm{ab}$ & $57,90 \mathrm{~d}$ \\
\hline & Zora & 60,48 & cde & 58,01 & $a b$ & 54,01 & $a b$ & 54,82 & $a b$ & $56,83 d$ \\
\hline & Neoplanta & 73,73 & $\mathrm{a}$ & 65,62 & $\mathrm{a}$ & 59,96 & $\mathrm{a}$ & 57,44 & $\mathrm{ab}$ & $64,19 a$ \\
\hline & P21T45 & 63,99 & bcd & 58,26 & $\mathrm{ab}$ & 55,32 & $\mathrm{ab}$ & 56,76 & $\mathrm{ab}$ & $58,58 \mathrm{~cd}$ \\
\hline & Celina & 65,78 & abcd & 61,40 & $\mathrm{ab}$ & 64,66 & $\mathrm{a}$ & 59,47 & $\mathrm{a}$ & $62,83 a b c$ \\
\hline & Adonai & 70,98 & $\mathrm{ab}$ & 65,03 & $\mathrm{a}$ & 58,07 & $\mathrm{a}$ & 57,88 & $\mathrm{ab}$ & $62,99 \mathrm{ab}$ \\
\hline & SED & 1,94 & & 2,09 & & 3,33 & & 1,65 & & $\operatorname{SED}(G)=1,37$ \\
\hline & MEAN ( C ) & 63,68 & a & 57,90 & $\mathrm{~b}$ & 57,04 & $\mathrm{~b}$ & 55,81 & $\mathrm{~b}$ & $\operatorname{SED}(C)=0,91$ \\
\hline
\end{tabular}

* At each time interval (days), values followed by the same letter, within each factor, are not significantly different according to LSD (P $\leq 0.05)$

Table 3: Seedling water content (WC \%) of soybean seedlings as affected by genotype (G) and $\mathrm{NaCl}$ concentration (C) at $7^{\text {th }}$ and $12^{\text {th }}$ day of salinity stress.

\begin{tabular}{|c|c|c|c|c|c|c|c|c|c|c|}
\hline \multirow{3}{*}{$\begin{array}{c}\text { Time (d) } \\
7\end{array}$} & \multirow{3}{*}{ Genotype (G) } & \multicolumn{8}{|c|}{$\mathrm{NaCl}$ Concentration $(\mathrm{mM})(\mathrm{C})$} & \multirow[b]{3}{*}{ MEAN ( G ) } \\
\hline & & \multicolumn{2}{|c|}{$\mathbf{0}$} & \multicolumn{2}{|c|}{50} & \multicolumn{2}{|c|}{100} & \multicolumn{2}{|c|}{200} & \\
\hline & & & & & & & & & & \\
\hline & PR92B63 & 70,66 & $\mathrm{ab}$ & 67,55 & $a b$ & 67,83 & $\mathrm{a}$ & 65,26 & $\mathrm{ab}$ & $67,82 \mathrm{abc}$ \\
\hline & PR92M35 & 66,58 & $\mathrm{~b}$ & 63,52 & c & 61,28 & c & 61,16 & $\mathrm{~b}$ & $63,14 \mathrm{e}$ \\
\hline & PR92M22 & 74,41 & $\mathrm{ab}$ & 64,82 & bc & 63,11 & bc & 61,62 & $\mathrm{ab}$ & 65,99bcde \\
\hline & PR91M10 & 65,63 & $\mathrm{~b}$ & 64,29 & bc & 63,41 & $\mathrm{bc}$ & 63,53 & $\mathrm{ab}$ & $64,21 \mathrm{de}$ \\
\hline & Zora & 72,21 & $\mathrm{ab}$ & 64,64 & bc & 62,84 & bc & 62,10 & $\mathrm{ab}$ & 65,45 cde \\
\hline & Neoplanta & 78,04 & $\mathrm{a}$ & 70,18 & $\mathrm{a}$ & 65,93 & $\mathrm{ab}$ & 60,77 & $\mathrm{ab}$ & $68,73 \mathrm{ab}$ \\
\hline
\end{tabular}




\section{International Journal of Environmental Sciences \& Natural Resources}

\begin{tabular}{|c|c|c|c|c|c|c|c|c|c|c|}
\hline & P21T45 & 80,92 & $\mathrm{a}$ & 66,02 & $\mathrm{bc}$ & 65,39 & $\mathrm{ab}$ & 66,76 & $\mathrm{a}$ & $69,77 a$ \\
\hline & Celina & 71,26 & $\mathrm{ab}$ & 65,47 & $\mathrm{bc}$ & 64,32 & $\mathrm{abc}$ & 63,45 & $\mathrm{ab}$ & $66,12 \mathrm{bcd}$ \\
\hline & Adonai & 74,63 & $\mathrm{ab}$ & 71,17 & a & 65,08 & $\mathrm{abc}$ & 59,89 & $\mathrm{ab}$ & 67,69abc \\
\hline & SED & 3,12 & & 1,12 & & 1,19 & & 1,79 & & $\operatorname{SED}(G)=0,91$ \\
\hline & MEAN ( C ) & 72,7 & $\mathrm{a}$ & 66,4 & $\mathrm{~b}$ & 64,35 & c & 62,73 & $\mathrm{~d}$ & $\operatorname{SED}(C)=0,61$ \\
\hline \multirow[t]{12}{*}{12} & & & & & & & & & & MEAN ( G ) \\
\hline & PR92B63 & 76,39 & bc & 71,76 & $\mathrm{~b}$ & 75,38 & a & 73,64 & $\mathrm{a}$ & $74,29 a b$ \\
\hline & PR92M35 & 76,92 & $\mathrm{bc}$ & 72,17 & $\mathrm{ab}$ & 69,83 & $\mathrm{~b}$ & 68,39 & $\mathrm{a}$ & $71,82 \mathrm{bc}$ \\
\hline & PR92M22 & 82,68 & $\mathrm{a}$ & 74,56 & $\mathrm{ab}$ & 69,75 & $\mathrm{~b}$ & 69,39 & $\mathrm{a}$ & $74,09 \mathrm{ab}$ \\
\hline & PR91M10 & 74,44 & c & 73,03 & $\mathrm{ab}$ & 69,69 & $\mathrm{~b}$ & 67,89 & $\mathrm{a}$ & $71,26 \mathrm{bc}$ \\
\hline & Zora & 80,13 & $a b$ & 74,87 & $\mathrm{ab}$ & 71,01 & $\mathrm{ab}$ & 70,67 & $\mathrm{a}$ & $74,17 \mathrm{ab}$ \\
\hline & Neoplanta & 83,18 & $\mathrm{a}$ & 79,19 & a & 71,31 & $\mathrm{ab}$ & 67,66 & $\mathrm{a}$ & $75,33 a$ \\
\hline & P21T45 & 74,58 & $\mathrm{c}$ & 70,69 & $\mathrm{~b}$ & 72,49 & $\mathrm{ab}$ & 72,67 & $\mathrm{a}$ & $72,61 \mathrm{bc}$ \\
\hline & Celina & 76,86 & bc & 70,81 & $\mathrm{~b}$ & 71,96 & $\mathrm{ab}$ & 69,85 & $\mathrm{a}$ & $72,37 \mathrm{bc}$ \\
\hline & Adonai & 82,28 & $\mathrm{a}$ & 79,14 & a & 71,07 & $\mathrm{ab}$ & 69,38 & $\mathrm{a}$ & $75,47 a$ \\
\hline & SED & 1,59 & & 2,15 & & 1,40 & & 1,61 & & $\operatorname{SED}(G)=0,80$ \\
\hline & MEAN ( C ) & 78,6 & $\mathrm{a}$ & 74,02 & $\mathrm{~b}$ & 71,39 & c & 69,94 & $\mathrm{~d}$ & $\operatorname{SED}(C)=0,54$ \\
\hline
\end{tabular}

*At each time interval (days), values followed by the same letter, within each factor, are not significantly different according to LSD (P $\leq 0.05)$.

Further, our findings indicated significant differences in postgerminative growth among genotypes and treatments (Figure 2 \& $3)$. As expected, the emergence of root and shoot tissues of stressed seedlings delayed proportionally to the stress level applied, as compared to control seedlings. In relation to growth potential, genotypes differed significantly both under control and stress conditions. Although the length of roots decreased proportionally to the stress level, varieties PR92M22, Celina, Neoplanta and Adonai showed the highest root length. At $200 \mathrm{mM} \mathrm{NaCl}$, even these varieties showed a significant decrease in root length, yet
PR92M22 and Celina exhibited the lowest decrease. Accordingly, growth of shoots was strongly inhibited as $\mathrm{NaCl}$ concentration increased, thus leading to a sharp reduction at $200 \mathrm{mM} \mathrm{NaCl}$. With respect to shoot length, Neoplanta, PR92M22, Celina and Adonai were the best performing varieties, nevertheless showing a drastic reduction in shoot length at 100 and $200 \mathrm{mM} \mathrm{NaCl}$. At the other end, PR92B63, PR91M10 and PR92M35 were incapable of shoot formation at $200 \mathrm{mM} \mathrm{NaCl}$. Despite the drastic effects of salt stress in growth potential, it did not lead to the emergence of seedlings with abnormal phenotype.

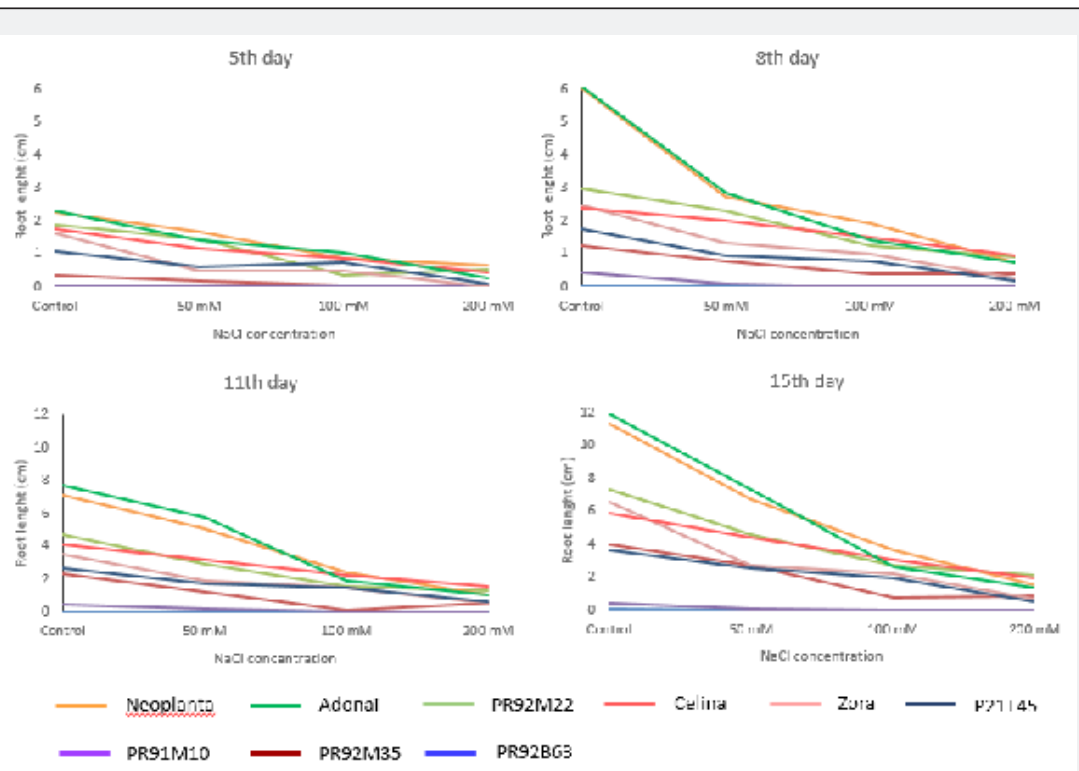

Figure 2: Root length $(\mathrm{cm})$ of nine soybean genotypes at different levels of salinity stress and at four time intervals. 


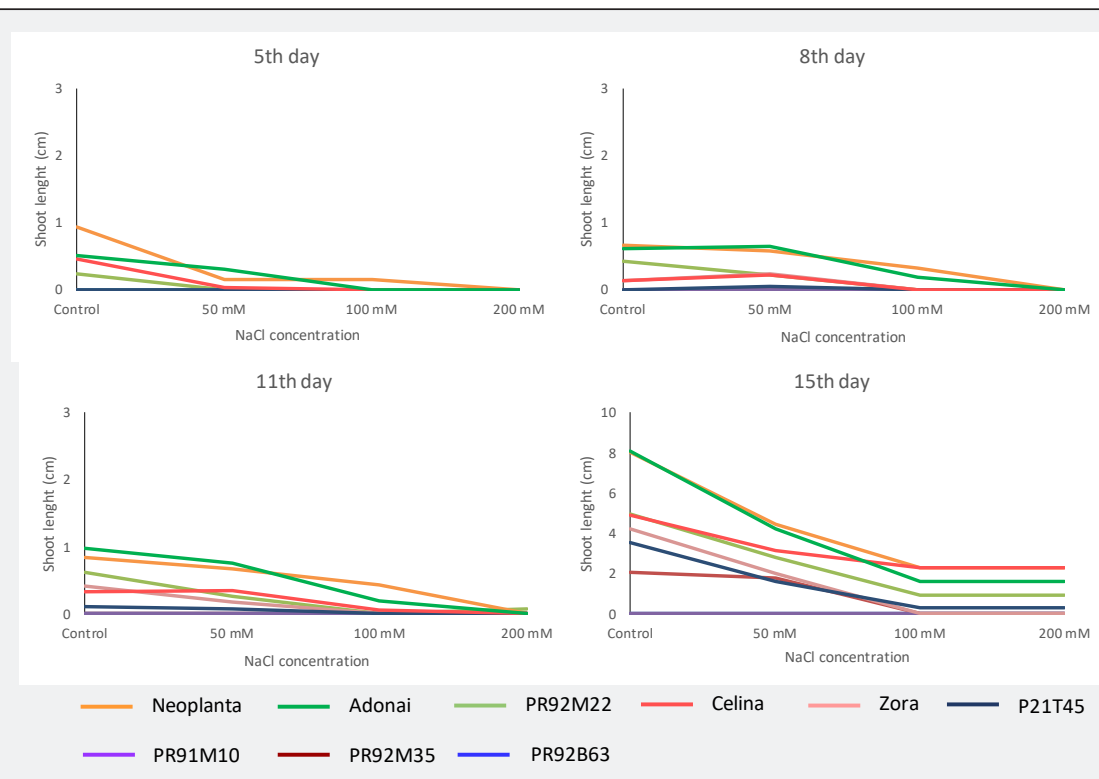

Figure 3: Shoot length $(\mathrm{cm})$ of nine soybean genotypes at different levels of salinity stress and at four time intervals.

\section{Discussion}

Plants grow in natural environments which are constantly changing while, at the same time, they are subjected to a complex set of abiotic and biotic stresses being the primary causes of crop loss worldwide. Among adverse environmental conditions, salinity is considered as one of the major limiting factors for plant growth, development and productivity, thus posing threats to crops' economic viability and food sufficiency worldwide. The effects of hypersalinity are mainly attributed to a low osmotic potential of soil solution, ion toxicity, nutritional imbalances and/or a combination of abovementioned factors. Salinity stress negatively impacts almost all developmental stages during the plant lifecycle, including seed germination as well as seedling establishment and subsequent growth [22]. Recent estimates project that $50 \%$ of total arable land will be affected by salinity by 2050 and, under stress conditions, the use of genotypes with genetic resistance will be the only means of enabling plant survival and ensuring minimization of yield losses encountered [23].

Although soybean's sensitivity to salinity maximizes during flowering and early grain development, as in most grain crops, salt stress adversely affects yield through inhibition or delay of seed germination and seedling growth [15-18]. Given that traits related to seed germination and seedling growth are salinity responsive traits, they have been proposed as valuable criteria for estimates of related genetic variation as well as for screening for salt tolerance at early growth stages. In this context, the present study pursued the identification of salt tolerant soybean genotypes based on seed germination and seedling growth potential under conditions of salinity stress.

Overall findings point to the conclusion that salinity significantly affects all traits associated with seed germination and seedling establishment, with the effects of stress being correlated to the stress level applied, thus maximizing at high stress level $(200 \mathrm{mM} \mathrm{NaCl})$. Such findings are consistent with previous reports and provide further evidence that, depending on stress level, salinity may be either inhibitive to germination, without loss of viability, or a delaying factor at stress levels where germination is not totally repressed [24-26]. In our study, although germination percentage was significantly affected in all genotypes, the analysis revealed the existence of considerable genetic variation for seed germination potential under salt stress conditions, with varieties Neoplanta and Adonai being classified as most tolerant at low and medium stress levels. At high stress level, all genotypes suffered significant decrease in germination rate, with the exception of Neoplanta which retained a high germination ability. These findings are indicative of the potential use of Neoplanta either for cultivation under conditions of hypersalinity or for exploitation as suitable germplasm in breeding programs specifically targeted at the improvement of salt tolerance in soybean.

In relation to post-germination growth under stress conditions, our findings underline that shoot and root length were substantially affected by increased salinity, with most profound effects being noted at high stress level. At this level, although shoot and root length were severely affected in all genotypes, genotypic differences were maximized leading to an array of responses ranging from moderate tissue elongation to total repression of tissue formation. To this respect, Neoplanta, Adonai, Celina, and PR92M22 exhibited the highest seedling growth potential under stress conditions.

Apart from the classification of genotypes according to their response to salt stress, this study underlines the possibility of using this approach for screening for salt tolerance at early growth stages, employing high stress levels and preferably combining 
them with germination percentage and data for root and shoot length. In this regard, root length reduction in response to salt stress has been previously proposed as an indicator of salinity tolerance at germination stage in soybean [27]. In contrast, the traits of seed water absorbance (WU) and seedling water content (WC) did not provide the possibility to accurately classify cultivars according to their performance, therefore indicating that they do not form suitable screening criteria for salt tolerance.

Conclusively, our findings underline the existence of large genetic variation related to salt tolerance at germination phase among the different soybean genotypes, while further suggesting the suitability of the employed approach to reveal genetic variation existing in soybean germplasm of unknown salt tolerance. Further, our findings provide strong evidence for the superiority of Neoplanta, Adonai, Celina, and PR92M22 in tolerating increased salinity while on the other hand, PR92B63 and PR91M10 were classified as salt-sensitive genotypes. Upon validation of these findings under conditions of salinity in the field, this screening approach may serve for selecting desirable germplasm material at early growth stages to be directly cultivated in saline soils or to be exploited in relative breeding programs.

\section{References}

1. Yan L (2008) Effect of salt stress on seed germination and seedling growth of three salinity plants. Pakistan Journal of Biological Sciences 11(9): 1268-1272.

2. Serrano R, Macia FC, Moreno V (1999) Genetic engineering of salt and drought tolerance with yeast regulatory genes. Scientia Horticulturae 78(1-4): 261-269.

3. Bewley JD, Black M (1982) Physiology and biochemistry of seeds in relation to germination. Vol 2, Springer, Berlin, Germany, pp. 13991401.

4. Murillo AB, Lopez AR, Kaya C, Larrinaga MJ, Flores HA (2002) Comparative effects of $\mathrm{NaCl}$ and polyethylene glycol on germination, emergence and seedling growth of cowpea. Journal of Agronomy and Crop Science 188(4): 235-247.

5. Khajeh HM, Powell AA, Bingham IJ (2003) The interaction between salinity stress and seed vigor during germination of soya bean seeds. Seed Science and Technology 31(3): 715-725.

6. Carillo P, Mastrolonardo G, Nacca F, Fuggi A (2005) Nitrate reductase in durum wheat seedlings as affected by nitrate nutrition and salinity. Functional Plant Biology 32(3): 209-219.

7. Sairam RK, Tyagi A (2004) Physiology and molecular biology of salinity stress tolerance in plants. Current Science 86(3): 407-421

8. Nasir Khan M, Siddiqui MH, Mohammad F, Masroor M, Khan A, et al. (2007) Salinity induced changes in growth, enzyme activities, photosynthesis, proline accumulation and yield in linseed genotypes. World Journal of Agricultural Sciences 3: 685-695.

9. Katerji N, Van Hoorn JW, Hamdy A, Mastrorilli M (2000) Salt tolerance classification of crops according to soil salinity and to water stress dry index. Agricultural Water Management 43(1): 99-109.

10. Ashraf M (1994) Genetic variation for salinity tolerance in spring wheat. Hereditas 120(2): 99-104.
11. Katerji N, Hoorn JWV, Hamdy A, Mastrorilli M (2003) Salinity effect on crop development and yield, analysis of salt tolerance according to several classification methods. Agricultural Water Management 62(1): $37-66$.

12. Chinusamy V, Jagendorf A, Zhu JK (2005) Understanding and improving salt tolerance in plants. Crop Science 45(2): 437-448.

13. Ashraf M, McNeilly T, Bradshaw AD (1987) Selection and heritability of tolerance to sodium chloride in four forage species. Crop Science 27(2): 232-234.

14. Kitajima K, Fenner M (2000) Ecology of seedling regeneration In: Fenner M (Ed.), Seeds: the ecology of regeneration in plant communities. ( $\left.2^{\text {nd }} e d n\right)$, CABI Publishing, Wallingford, UK, pp. 331-359.

15. Kondetti P, Jawali N, Apte SK, Shitole MG (2012) Salt tolerance in Indian soybean (Glycine $\max (\mathrm{L}$.) Merrill) varieties at germination and early seedling growth. Annals of Biological Research 3: 1489-1498.

16. Kandil AA, Sharief AE, Ahmed KhR (2015) Performance of some soybean (Glycine max (L.) Merrill) cultivars under salinity stress to germination characters. International Journal of Agronomy and Agricultural Research 6(3): 48-56.

17. Dhairyasheel B, Patil B, Sharad B (2015) Influence of NaCl-mediated salinity stress on lipid peroxidation in germinating seeds of soybean. International Journal of Pharma and Bio Sciences 6(1): 549-552.

18. Hamayun M, Hussain A, Khan SA, Irshad M, Khan AL, et al. (2015) Kinetin modulates physio-hormonal attributes and isoflavone contents of soybean grown under salinity stress. Frontiers in Plant Science 6(e0012): 377 .

19. Mujeeb-ur-Rahman, Soomro UA, Zahoor-ul-Haq M, Gul S (2008) Effects of $\mathrm{NaCl}$ salinity on wheat (Triticum aestivum L.) cultivars. World Journal of Agricultural Sciences 4(3): 398-403.

20. Black M, Pritchard H (2002) Desiccation and survival in plants drying without dying. CABI, New York, USA, pp. 93-110.

21. Ahmadvand G, Soleimani F, Saadatian B, Pouya M (2012) Effect of seed priming with potassium nitrate on germination and emergence traits of two soybean cultivars under salinity stress condition. AmericanEurasian Journal of Agricultural and Environmental Sciences 12(6): 769-774.

22. Zhu JK (2016) Abiotic Stress Signaling and Responses in Plants. Cell 167(2): 313-324.

23. Butcher K, Wick AF, Desutter T, Chatterjee A, Harmon JP (2016) Soil salinity: A threat to global food security. Agronomy Journal 108(6): 2189-2200.

24. Gulzar S, Khan MA (2001) Seed germination of a halophytic grass Aeluropus lagopoides. Annals of Botany 87(3): 319-324.

25. Buchade JY, Karadge BA (2014) Effect of $\mathrm{NaCl}$ on seed germination in five legume crops. Journal of Advanced Scientific Research 5(2): 56-58.

26. Ouji A, Safia EB, Mouelhi M, Younes MB, Kharrat M (2015) Effect of salinity stress on germination of five Tunisian Lentil (Lens culinaris L.) genotypes. European Scientific Journal 11(21): 63-75.

27. Agarwal N, Kumar A, Agarwal S, Singh A (2015) Evaluation of soybean (Glycine max L.) cultivars under salinity stress during early vegetative growth. International Journal of Current Microbiology and Applied Sciences 4(2): 123-134. 
(C) This work is licensed under Creative BY Commons Attribution 4.0 License

DOI:10.19080/IJESNR.2021.27.556210
Your next submission with Juniper Publishers will reach you the below assets

- Quality Editorial service

- Swift Peer Review

- Reprints availability

- E-prints Service

- Manuscript Podcast for convenient understanding

- Global attainment for your research

- Manuscript accessibility in different formats ( Pdf, E-pub, Full Text, Audio)

- Unceasing customer service

Track the below URL for one-step submission https://juniperpublishers.com/online-submission.php 